Acta Crystallographica Section F

Structural Biology

Communications

ISSN 2053-230X

Jaeok Park, ${ }^{a}$ Yih-Shyan Lin, ${ }^{b}$ Youla S. Tsantrizos ${ }^{a, b, c}$ and Albert M. Berghuis ${ }^{a, c, d_{*}}$

${ }^{a}$ Department of Biochemistry, McGill University, 3655 Promenade Sir William Osler, Montreal, QC H3G 1Y6, Canada, 'b Department of Chemistry, McGill University, 801 Rue Sherbrooke Ouest, Montreal, QC H3A 0B8, Canada, ' ${ }^{\mathbf{C}}$ roupe de Recherche Axé sur la Structure des Protéines, McGill University, 3649 Promenade Sir William Osler, Montreal, QC H3G 0B1, Canada, and dDepartment of Microbiology and Immunology, McGill University, 3775 Rue University, Montreal, QC H3A 2B4, Canada

Correspondence e-mail: albert.berghuis@mcgill.ca

Received 18 December 2013

Accepted 29 January 2014

PDB reference: farnesyl pyrophosphate synthase, complex with YSO470 and two molecules of inorganic phosphate, $4 \mathrm{lfv}$

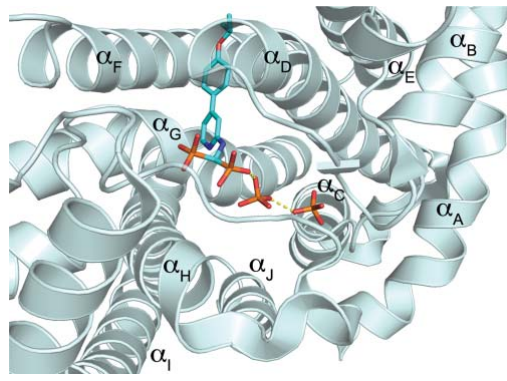

\section{Structure of human farnesyl pyrophosphate synthase in complex with an aminopyridine bisphosphonate and two molecules of inorganic phosphate}

Human farnesyl pyrophosphate synthase (hFPPS) produces farnesyl pyrophosphate, an isoprenoid essential for a variety of cellular processes. The enzyme has been well established as the molecular target of the nitrogen-containing bisphosphonates (N-BPs), which are best known for their antiresorptive effects in bone but are also known for their anticancer properties. Crystal structures of hFPPS in ternary complexes with a novel bisphosphonate, YS0470, and the secondary ligands inorganic phosphate $\left(\mathrm{P}_{\mathrm{i}}\right)$, inorganic pyrophosphate $\left(\mathrm{PP}_{\mathrm{i}}\right)$ and isopentenyl pyrophosphate (IPP) have recently been reported. Only the cobinding of the bisphosphonate with either $\mathrm{PP}_{\mathrm{i}}$ or IPP resulted in the full closure of the C-terminal tail of the enzyme, a conformational change that is required for catalysis and that is also responsible for the potent in vivo efficacy of N-BPs. In the present communication, a co-crystal structure of hFPPS in complex with YS0470 and two molecules of $\mathrm{P}_{\mathrm{i}}$ is reported. The unusually close proximity between these ligands, which was confirmed by anomalous diffraction data, suggests that they interact with one another, with their anionic charges neutralized in their bound state. The structure also showed the tail of the enzyme to be fully disordered, indicating that simultaneous binding of two $\mathrm{P}_{\mathrm{i}}$ molecules with a bisphosphonate cannot induce the tail-closing conformational change in hFPPS. Examination of homologous FPPSs suggested that this liganddependent tail closure is only conserved in the mammalian proteins. The prevalence of $\mathrm{P}_{\mathrm{i}}$-bound hFPPS structures in the PDB raises a question regarding the in vivo relevance of $\mathrm{P}_{\mathrm{i}}$ binding to the function of the enzyme.

\section{Introduction}

Human farnesyl pyrophosphate synthase (hFPPS) occupies the first branching point in the mevalonate pathway and carries out the elongation of dimethylallyl pyrophosphate (DMAPP) to geranyl pyrophosphate (GPP) and then to farnesyl pyrophosphate (FPP) by successively condensing two molecules of isopentenyl pyrophosphate (IPP). FPP is absolutely required for post-translational modification (i.e. prenylation) of small signalling GTPases, which is essential for their subcellular localization and function (McTaggart, 2006). Given the role of small GTPases as regulators of fundamental cellular processes, blocking their prenylation via hFPPS inhibition serves as a useful and effective means of pharmacological intervention. Currently, nitrogen-containing bisphosphonates (N-BPs), such as zoledronate and risedronate, comprise the only class of clinically approved drugs targeting hFPPS. These drugs have been widely used against bone-resorption disorders, but are also gaining a great deal of interest for their anticancer properties (Koul et al., 2012).

The mechanism by which N-BPs inhibit hFPPS has been well characterized by X-ray crystallographic studies (Kavanagh et al., 2006; Rondeau et al., 2006). They bind to the DMAPP/GPP subpocket of the active site, mimicking and competing with these substrates. The inhibition also involves ligand-induced conformational changes in the enzyme. Occupancy of the DMAPP/GPP subpocket results in a rigid-body movement that closes this binding site and shapes the IPP subpocket (i.e. from the open to the partially closed state). Subsequent IPP binding induces full structuring of the four-residue C-terminal tail, which in turn closes the IPP subpocket and sequesters the active site from the solvent environment (i.e. from the partially closed to the fully closed state). While hFPPS cycles 
Table 1

Data-collection and structure-refinement statistics.

Values in parentheses are for the outer shell.

\begin{tabular}{|c|c|c|}
\hline & $\begin{array}{l}\text { Data set } 1 \\
\text { (synchrotron) }\end{array}$ & $\begin{array}{l}\text { Data set } 2 \\
\text { (home source) }\end{array}$ \\
\hline \multicolumn{3}{|l|}{ Data collection } \\
\hline Wavelength (§̊) & 0.97949 & 1.5418 \\
\hline Space group & $P 4_{1} 2_{1} 2$ & $P 4_{1} 2_{1} 2$ \\
\hline Unit-cell parameters $\left(\AA{ }^{\circ}{ }^{\circ}\right)$ & $\begin{array}{c}a=b=111.0, c=67.0, \\
\alpha=\beta=\gamma=90\end{array}$ & $\begin{array}{c}a=b=111.0, c=69.9, \\
\alpha=\beta=\gamma=90\end{array}$ \\
\hline No. of molecules in asymmetric unit & 1 & 1 \\
\hline Matthews coefficient $V_{\mathrm{M}}\left(\AA^{3} \mathrm{Da}^{-1}\right)$ & 2.39 & 2.50 \\
\hline Solvent content $(\%)$ & 48.62 & 50.74 \\
\hline Resolution $(\AA)$ & $67.04-2.00(2.05-2.00)$ & $39.24-2.46(2.52-2.46)$ \\
\hline No. of unique reflections & $28844(2083)$ & $16393(1144)$ \\
\hline Mean $\langle I / \sigma(I)\rangle$ & $28.8(7.4)$ & $49.2(3.8)$ \\
\hline Completeness $(\%)$ & $99.7(98.8)$ & $98.7(87.5)$ \\
\hline Multiplicity & $14.3(14.3)$ & $13.2(2.7)$ \\
\hline$R_{\text {merge }}$ & $0.070(0.498)$ & $0.054(0.389) \dagger$ \\
\hline \multicolumn{3}{|l|}{ Structure refinement } \\
\hline Resolution range $(\AA)$ & $51.03-2.00(2.05-2.00)$ & \\
\hline No. of reflections used, working set & $27134(1882)$ & \\
\hline No. of reflections used, test set & $1454(110)$ & \\
\hline No. of protein atoms in the model & 2728 & \\
\hline No. of water atoms in the model & 153 & \\
\hline No. of other atoms in the model & 40 & \\
\hline Overall average $B$ factor $\left(\AA^{2}\right)$ & 35.0 & \\
\hline Final $R_{\text {work }}$ & $0.177(0.202)$ & \\
\hline Final $R_{\text {free }}$ & $0.218(0.265)$ & \\
\hline R.m.s.d., bond lengths ( & 0.019 & \\
\hline R.m.s.d., bond angles $\left({ }^{\circ}\right)$ & 1.923 & \\
\hline \multicolumn{3}{|c|}{ Residues in Ramachandran regions (\%) } \\
\hline Favoured region & 98.5 & \\
\hline Allowed region & 1.5 & \\
\hline Outlier region & 0 & \\
\hline
\end{tabular}

$\dagger$ When merged.

through these conformational changes during catalysis, with chemically stable N-BPs the ternary enzyme complex becomes locked in the fully closed state. In this conformation, direct competition between the deeply bound inhibitor and DMAPP/GPP is impossible, and thus N-BP binding is considered to be nearly irreversible. The potent in vivo efficacy of N-BP drugs is therefore thought to arise in part from the stabilization of the enzyme-inhibitor complex by binding of the accumulating substrate IPP.

Despite the importance of the C-terminal tail closure in hFPPS, the mechanistic details of this conformational change have remained largely uncharacterized. By determining and analyzing crystal structures of hFPPS in ternary complexes with a novel bisphosphonate inhibitor, YS0470, and the secondary ligands inorganic phosphate $\left(\mathrm{P}_{\mathrm{i}}\right)$, inorganic pyrophosphate $\left(\mathrm{PP}_{\mathrm{i}}\right)$ and IPP, we recently identified the key residues and interactions responsible for the tail closure of the enzyme (Park et al., 2012). The secondary ligands were introduced by soaking in this study, and only the binding of $\mathrm{PP}_{\mathrm{i}}$ or IPP induced the full structuring of the C-terminal tail of the enzyme. More recently, we solved a crystal structure of hFPPS obtained under different conditions that exhibited unusual electron density in the second substrate-binding site. Analysis of anomalous diffraction data from an isomorphous crystal has allowed us to unambiguously identify the ligands bound at this site. We thus report in this communication a co-crystal structure of hFPPS in complex with YS0470 and two molecules of $\mathrm{P}_{\mathrm{i}}$.

\section{Materials and methods}

\subsection{Preparation of the protein and inhibitor samples}

The expression and purification of hFPPS, as well as the synthesis of YS0470, have been described in a previous report (Lin et al., 2012).
Table 2

PDB structures of hFPPS in complex with the bisphosphonate YS0470.

\begin{tabular}{|c|c|c|c|c|}
\hline $\begin{array}{l}\text { PDB code } \\
\text { (reference) }\end{array}$ & $\begin{array}{l}\text { Resolution } \\
(\AA)\end{array}$ & Ligands & $\begin{array}{l}\text { R.m.s.d. } \dagger \\
(\AA)\end{array}$ & $\begin{array}{l}\text { Overall } \\
\text { conformation }\end{array}$ \\
\hline 4dem (Lin et al., 2012) & 1.85 & YS0470末 & 0.17 & Partially closed \\
\hline 4h5c (Park et al., 2012) & 2.02 & YS0470, $P_{i}$ & 0.17 & Partially closed \\
\hline 4h5d (Park et al., 2012) & 2.02 & $\mathrm{YS} 0470, \mathrm{PP}_{\mathrm{i}}$ & 0.29 & Fully closed \\
\hline 4h5e (Park et al., 2012) & 2.05 & YS0470, IPP & 0.25 & Fully closed \\
\hline 4lfv (this work) & 2.00 & YS0470, $2 \mathrm{P}_{\mathrm{i}}$ & - & Partially closed \\
\hline
\end{tabular}

$\dagger$ Structure superposition was performed with SSM based on $335 \mathrm{C}^{\alpha}$ atoms. $\neq$ Bisphosphonate binding occurs via metal chelation involving three $\mathrm{Mg}$ ions, which are not included in this table.

\subsection{Crystallization}

Compound YS0470 was prepared as a $100 \mathrm{~m} M$ solution in $100 \mathrm{~m} M$ Tris- $\mathrm{HCl} \mathrm{pH} 7.5$, and $\mathrm{MgCl}_{2}$ was prepared as a $100 \mathrm{~m} M$ aqueous solution. These solutions were added to the hFPPS sample to give final concentrations of $1 \mathrm{~m} M$ inhibitor, $1.5 \mathrm{~m} M \mathrm{MgCl}_{2}$ and $0.25 \mathrm{~m} M$ $\left(10 \mathrm{mg} \mathrm{ml}^{-1}\right)$ protein. Crystals suitable for X-ray diffraction were obtained at $295 \mathrm{~K}$ by vapour diffusion in a sitting drop composed of $1 \mu \mathrm{l}$ inhibitor $/ \mathrm{MgCl}_{2} /$ protein mixture, $1 \mu \mathrm{l}$ crystallization solution (30\% PEG 400, $2 \mathrm{M}$ ammonium phosphate, $0.2 \mathrm{M} \mathrm{MgCl}_{2}, 0.1 \mathrm{M}$ HEPES pH 7.5) and $0.5 \mu \mathrm{l}$ seed stock. The seed stock was prepared with a Seed Bead kit (Hampton Research) using a crystal grown in a sitting drop consisting of $1 \mu \mathrm{l}$ ligand-free protein sample $\left(10 \mathrm{mg} \mathrm{ml}^{-1}\right)$ and $1 \mu \mathrm{l}$ crystallization solution $(2.0 \mathrm{M}$ ammonium phosphate, $0.1 \mathrm{M}$ Tris- $\mathrm{HCl} \mathrm{pH}$ 8.5).

\subsection{Data collection, processing and structure refinement}

For structure determination, diffraction data were collected from a single crystal at $100 \mathrm{~K}$ using synchrotron radiation (Canadian Light Source, Saskatoon, SK, Canada) and a Rayonix MX300 CCD detector. For exploiting the anomalous signal from $\mathrm{P}$ atoms, additional data were collected at home from another single crystal using a MicroMax-007 HF generator (Rigaku) and a Saturn 944+ CCD detector (Rigaku). Both data sets were processed with the xia2 package (Winter et al., 2013); for the home-source data the Friedel mates were not merged together, unlike for the synchrotron data. The structure model was initially built by a difference Fourier method with a ligand/solvent-omitted starting model generated from PDB entry 4h5d (Park et al., 2012). The model was improved through iterative rounds of manual and automated refinement with Coot (Emsley et al., 2010) and REFMAC5 (Murshudov et al., 2011). The final model was deposited in the Protein Data Bank (PDB entry 4lfv). Data-collection and refinement statistics are presented in Table 1.

\subsection{Anomalous density calculation}

An anomalous density map was calculated from the home-source data with the programs SHELXC (Sheldrick, 2010) and ANODE (Thorn \& Sheldrick, 2011). The phase information used in this calculation was obtained from the final structure model refined against the synchrotron data.

\section{Results and discussion}

\subsection{Overall structure}

The overall fold of the new structure (PDB entry 4lfv) is very similar to those of the previously described hFPPS-YS0470 complexes, as indicated by the r.m.s.d.s for superposition in Table 2. It is noteworthy that the r.m.s.d. values are lower with the secondary 
ligand-free (hFPPS-YS0470) and $\mathrm{P}_{\mathrm{i}}$-bound (hFPPS-YS0470- $\mathrm{P}_{\mathrm{i}}$ ) forms, which are in the partially closed conformation. This observation is consistent with the finding that the new complex is also in the partially closed conformation, the details of which we will discuss below.

\subsection{DMAPP/GPP subpocket and bisphosphonate binding}

The structure of the DMAPP/GPP subpocket is essentially identical in all of the reported YS0470-bound hFPPS complexes, including the present one. The interactions between the bisphosphonate and the protein have been discussed previously (De Schutter et al., 2012; Lin et al., 2012; Park et al., 2012).

\subsection{IPP subpocket and secondary ligands}

The initial density map produced by Fourier synthesis, phased with only protein atoms, indicated the presence of two ligands in the IPP subpocket. We could readily deduce the identity of one (density A in Fig. 1a) as $\mathrm{P}_{\mathrm{i}}$ based on the shape of its electron density as well as its location as a known $\mathrm{P}_{\mathrm{i}}$-binding site (Park et al., 2012). The most likely candidate for the second ligand was also $\mathrm{P}_{\mathrm{i}}$, given the electron-density contour (density B in Fig. 1a) and the composition of the crystallization mother liquor (see $\$ 2.2$ ). However, the proximity of the second $\mathrm{P}_{\mathrm{i}}$ to both the first $\mathrm{P}_{\mathrm{i}}$ and the bisphosphonate group of YS0470 (closest atomic distances of 2.3 and $2.5 \AA$, respectively) was puzzling, since all three of these ions should be negatively charged at the given $\mathrm{pH}$ and thus be subject to electrostatic repulsion.

In order to verify the identity of the second $\mathrm{P}_{\mathrm{i}}$, we collected a second data set in which the anomalous signal was preserved during processing. With $\mathrm{Cu} K \alpha$ radiation $(\lambda=1.5418 \AA$ ) $\mathrm{P}$ atoms displayed a measurable anomalous signal: the anomalous scattering contribution $\left(f^{\prime \prime}\right)$ of phosphorus is appreciable at this wavelength ( 0.45 electron units), although its X-ray absorption edge lies at a much longer wavelength ( $\lambda=5.7788 \AA$; Brennan \& Cowan, 1992). As a result, an

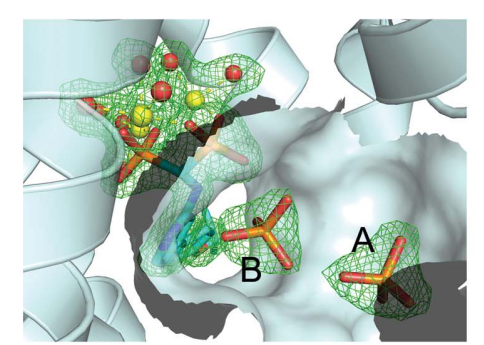

(a)

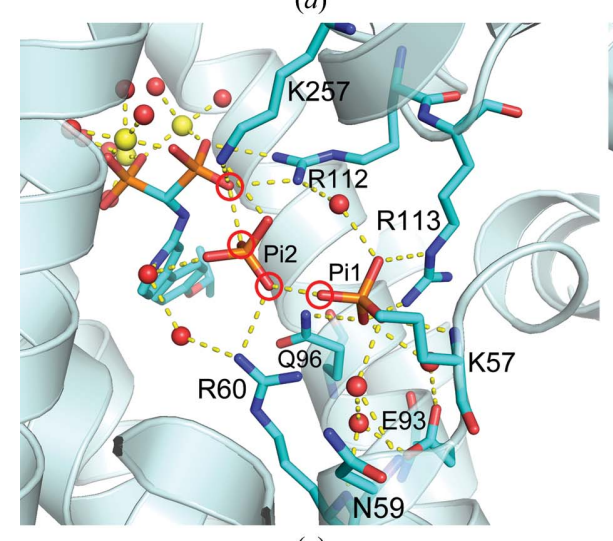

(c)

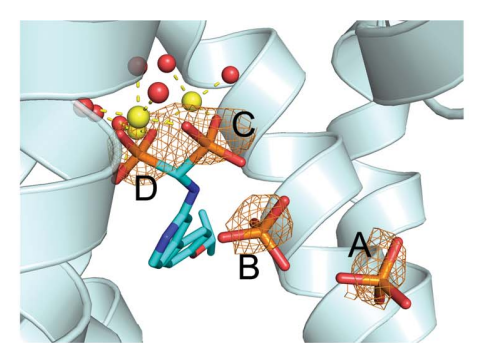

(b)

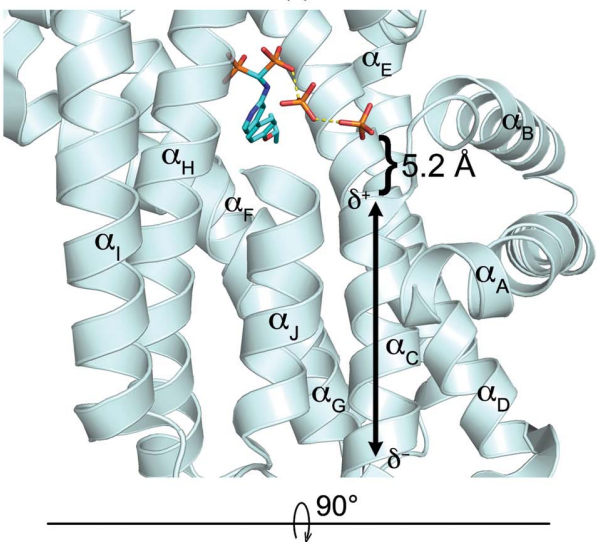

$\overbrace{}^{90^{\circ}}$

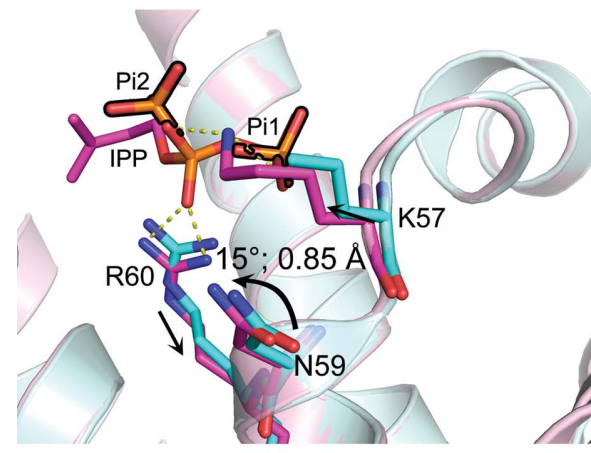

(e)

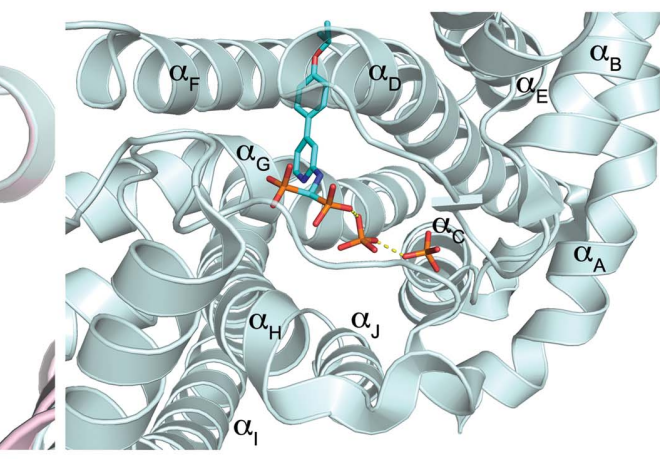

(d)

Figure 1

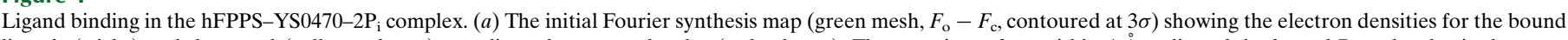

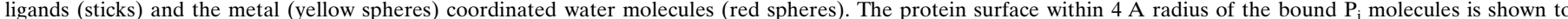

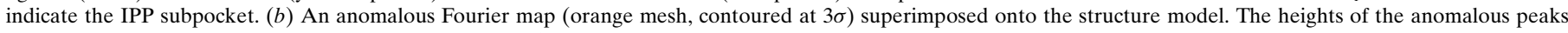

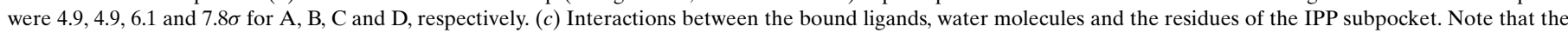

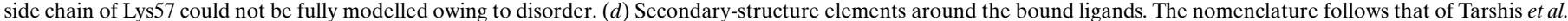

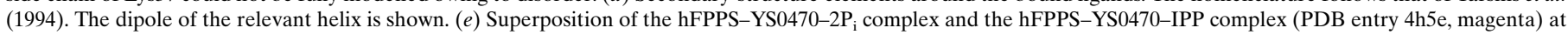

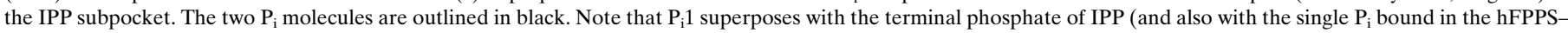
YS0470- $\mathrm{P}_{\mathrm{i}}$ complex; not shown). 
anomalous Fourier map calculated from the anomalous data set clearly demonstrated peaks that superposed on the $\mathrm{P}$ atoms of the first $\mathrm{P}_{\mathrm{i}}$ and YS0470 in our structure model, as well as that of the second $\mathrm{P}_{\mathrm{i}}$, thus confirming its identity (Fig. $1 b$ ).

The close proximity between the bisphosphonate and phosphate ligands suggests that their molecular charges may be neutralized by the surrounding ions and protons either on the nearby residues or the ligands. In addition to three $\mathrm{Mg}$ ions chelated to the bisphosphonate, the residues Arg60, Arg112, Arg113 and Lys257 are likely contributors, forming direct contacts with these ligands (Fig. 1c). Furthermore, the helix dipole of $\alpha_{\mathrm{C}}$ may also play a role in this regard, dissipating some of the charge on the first $\mathrm{P}_{\mathrm{i}}$ (Fig. 1d). Phosphate moieties frequently bind to the amino-termini of protein helices, typically at a distance of $3-5 \AA$, owing to the electric field generated by the helix backbone (Hol et al., 1978). Although our crystallographic data do not provide information regarding the protonation state of the ligands, the geometry and distances between the four interacting $\mathrm{O}$ atoms of the bisphosphonate and $\mathrm{P}_{\mathrm{i}}$ (circled in red in Fig. 1c) suggest that they form hydrogen bonds, with two of them being protonated.

As mentioned above, the enzyme-ligand complex reported here (hFPPS-YS0470-2 $\mathrm{P}_{\mathrm{i}}$ ) is in the partially closed state, like the hFPPSYS0470 and hFPPS-YS0470-P $\mathrm{P}_{\mathrm{i}}$ complexes. The conformational difference between the partially closed state and the fully closed state (as observed with the hFPPS-YS0470-PP ${ }_{\mathrm{i}}$ and hFPPS-YS0470-IPP complexes) is not overtly pronounced in the IPP subpocket: the $\alpha$-phosphate of the bound IPP (or the equivalent phosphate of the $\mathrm{PP}_{\mathrm{i}}$ ) attracts and structures Lys57 while pushing back Arg60; the displacements of these residues by less than $1 \AA$ result in a $\sim 15^{\circ}$ rotation of Asn59 (Fig. 1e). The second $\mathrm{P}_{\mathrm{i}}$ in the new complex, in comparison, is bound too distantly to interact with Lys57 ( $\mathrm{P}_{\mathrm{i}} 2$; Fig. 1e)

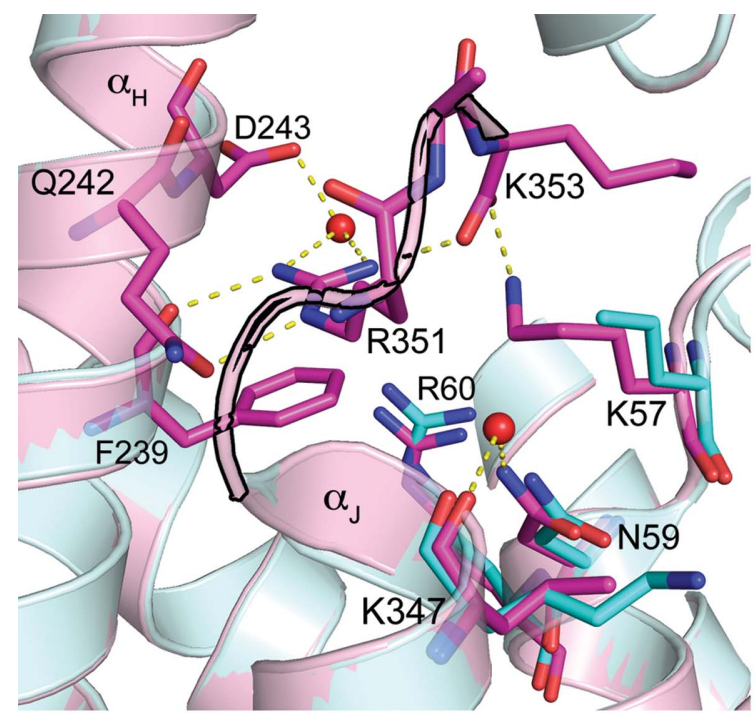

(a)

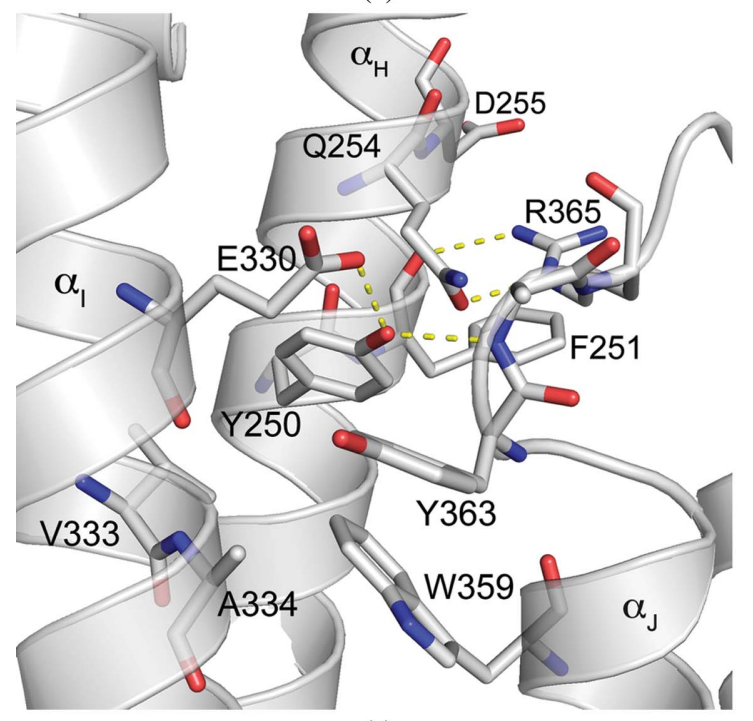

(c)

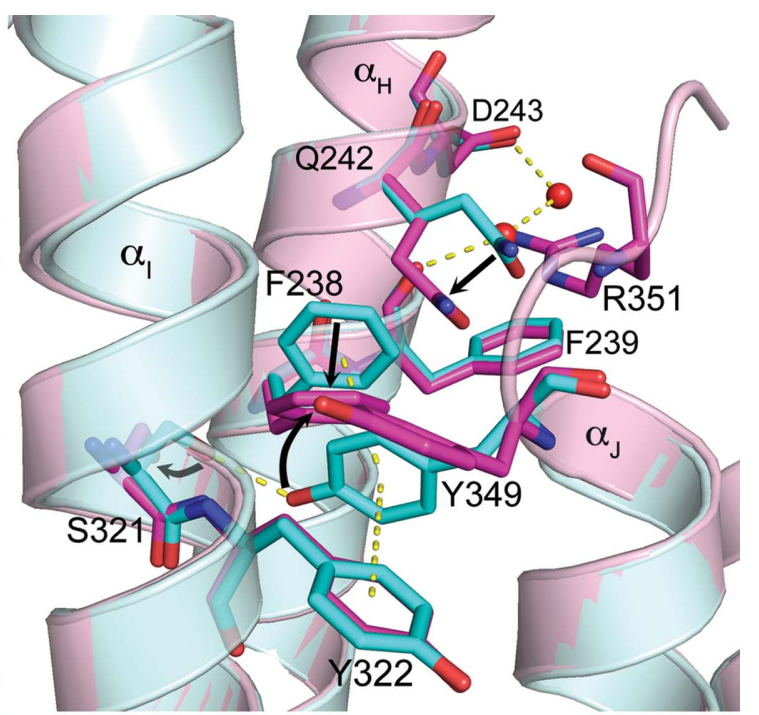

(b)

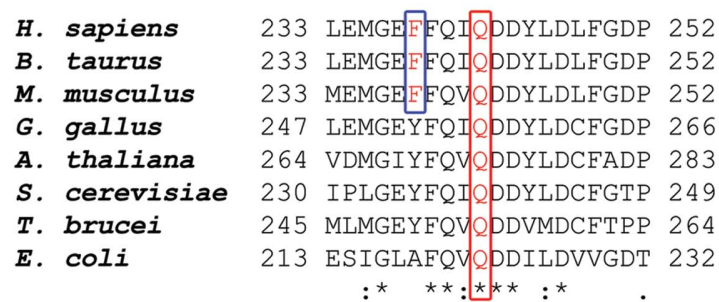

321 SYSHIMALIEQYAAP--LPPAVFLGLARKIYKRRK 353 321 SYNHIMGLIEQYAAP--LPPAIFLGLAQKIY $\mid R K K$ 321 SYNRLKSLIEQCSAP--LPPSIFMELANKIY RRK 353 334 SYRRLQELIEKHSNR--LPKEIFLGLAQKIYRRRK 367 352 SYEKLTGAIEGHQSK--AIQAVLKSFLAKIYARRK 384

318 IAKDLKAKISQVDESRGFKADVLTAFLNKVY IR SK 352 333 VAEQVKELIEKLRLCSPGFAASVETLWGKTYKRRK 367 272 --QSLKQLAEQSLDT-----SALEALADYIIORNK 299

$(d)$

Figure 2

Residues of the FPPS tail closure. (a) Tail closure induced by IPP binding. The hFPPS-YS0470-2P $\mathrm{i}_{\mathrm{i}}$ complex (cyan) and the hFPPS-YS0470-IPP complex (magenta) are superposed, with the bound ligands omitted for clarity. Note that the hFPPS-YS0470-2 $\mathrm{P}_{\mathrm{i}}$ complex is missing the C-terminal tail, which in the hFPPS-YS0470-IPP complex (highlighted in black) closes over the active-site entrance. The structures are shown in approximately the same orientation as in Fig. 1(e) (compare residues Lys57, Asn59 and Arg60). (b) The conformational change cascade required for the tail closure. In the absence of bound IPP/PP, , Tyr349 is trapped in the 'off' conformation by $\pi$-stacking and hydrogen-bond interactions. The 'off' conformations of Tyr349, Phe238 and Gln242 (cyan) prohibit the 'on' conformations of Phe238, Gln242 and Arg351 (magenta), respectively, by steric hindrance. (c) A second ligand-free T. brucei FPPS complex (PDB entry 3dyh, white) with its ordered C-terminal tail. ( $d$ ) Conserved residues of the FPPS tail closure. Sequence alignment was carried out with ClustalX (Larkin et al., 2007). 
and thus cannot bring about the above conformational changes. Although subtle, these changes are solely responsible for the subsequent ordering and closing of the C-terminal tail in hFPPS.

\subsection{C-terminal tail closure}

Previously, we observed electron density suggesting partial ordering of the C-terminal tail in the hFPPS-YS0470 and hFPPSYS0470- $\mathrm{P}_{\mathrm{i}}$ complexes, and could refine only the backbone atoms of the tail in the structure models (Lin et al., 2012; Park et al., 2012). The corresponding electron density was weaker for the hFPPS-YS0470$2 \mathrm{P}_{\mathrm{i}}$ complex, and the four C-terminal residues could not be built into our new structure model. In contrast, the C-terminal tail in the hFPPS-YS0470-PP ${ }_{i}$ and hFPPS-YS0470-IPP complexes was shown to be fully structured (Park et al., 2012). The full structuring of the C-terminal tail is most notably characterized by ordering of the Arg351 side chain, which anchors itself to helix $\alpha_{\mathrm{H}}$ and also forms a salt bridge with the terminal residue Lys353, thereby providing the otherwise flexible tail with rigidity (Fig. $2 a$ ).

We also showed previously that the anchoring of Arg351 requires a series of preceding conformational changes in the residues Gln242, Phe238 and Tyr349 (Fig. 2b; Park et al., 2012). Tyr349 is likely to function as a safety switch to prevent futile tail closure in the absence of bound IPP: when it is locked in the 'off' position, the downstream conformational changes are prohibited by steric hindrance (Fig. $2 b$ ). Examination of homologous FPPS proteins provides interesting perspectives regarding this control mechanism. In Trypanosoma brucei FPPS, Tyr363 cannot assume the 'off' conformation observed for its human counterpart owing to a neighbouring tryptophan (Trp359) hindering such a conformation (Fig. 2c). In addition, Tyr250, which corresponds to Phe238 in the human enzyme, is trapped in the 'on' conformation by two adjacent residues via hydrogen bonds (Fig. 2c). Not surprisingly, the C-terminal tail of T. brucei FPPS is fully structured in all nine structures available in the PDB, regardless of the presence of bound IPP (e.g. PDB entry 3dyh; Fig. $2 c$; see Supplementary Table $\mathrm{S} 1^{\mathbf{1}}$ for a complete list). T. cruzi FPPS also shows similar conformations for the equivalent residues and the C-terminal tail (12 structures in the PDB; Supplementary Table S1), although it has a histidine residue at the position of the switch tyrosine. Interestingly, the switch tyrosine and the three interacting residues (i.e. Phe238, Ser321 and Tyr322 in hFPPS) are fully conserved only in mammalian species, in contrast to the indispensable glutamine and arginine residues (Fig. $2 d$ ). This observation suggests that the tail-closure control feature in FPPS is exclusively characteristic of the mammalian order. A putative mechanism by which the subtle IPP/ $/ \mathrm{PP}_{\mathrm{i}}$-induced conformational change in the IPP subpocket is translated into such a drastic movement of Tyr349 (i.e. $>70^{\circ}$ rotation of the side chain), thus allowing the full closure of the C-terminal tail in hFPPS, has been described in detail previously (Park et al., 2012).

\subsection{Significance of $P_{i}$ binding}

A retrospective examination of all of the previously determined structures of hFPPS in the PDB (30 entries, excluding our own entries) identified 19 structures that have bound $P_{i}$ in the IPP subpocket (Supplementary Table S2). Other non-substrate ligands shown to bind at this site include inorganic pyrophosphate and sulfate. It is presently unknown whether the binding of $P_{i}$ or other negatively charged ions to hFPPS is a physiologically relevant event

\footnotetext{
${ }^{1}$ Supporting information has been deposited in the IUCr electronic archive (Reference: HV5250).
}

or strictly a crystallization artefact. However, it is possible that $\mathrm{P}_{\mathrm{i}}$ binding occurs in vivo, especially with its millimolar-scale intracellular concentration (Bevington et al., 1986). In such a case $\mathrm{P}_{\mathrm{i}}$ would inhibit the enzyme by competing with the substrate IPP, the intracellular concentration of which, in contrast, is in the picomolar range (Mönkkönen et al., 2008). Although the kinetics have not been studied in detail, in vitro inhibition of FPPS by a high concentration of $P_{i}$ was reported a long time ago (Holloway \& Popják, 1967). Interestingly, elevated levels of $P_{i}$ produce antiproliferative effects in multiple cancer cell lines by an as yet unknown mechanism involving reduced ERK1/2 phosphorylation (Spina, Sapio et al., 2013; Spina, Sorvillo et al., 2013). As downregulation of ERK phosphorylation is also a cellular hallmark of hFPPS inhibition (Lin et al., 2012), it is conceivable that $P_{i}$ elicits the antiproliferative effects (in part) by inhibiting hFPPS. However, this hypothesis is at present unproven.

\section{Conclusion}

The crystal structure of hFPPS reported in this communication demonstrates that the IPP subpocket of the enzyme can accommodate two molecules of $\mathrm{P}_{\mathrm{i}}$ simultaneously. The binding of two $\mathrm{P}_{\mathrm{i}}$ molecules did not induce the $\mathrm{C}$-terminal tail closure in the enzyme, unlike that of IPP or $\mathrm{PP}_{\mathrm{i}}$. This ligand-controlled conformational change is likely to be conserved only in mammalian FPPS. The possibility that $\mathrm{P}_{\mathrm{i}}$ serves as a modulator of hFPPS function in vivo warrants future studies. Our work also showed that the new program $A N O D E$ is effective in analyzing weak anomalous data and can be useful in identifying unknown ligands.

We thank the members of the Berghuis laboratory for their help and support, especially Mr Dmitry Rodionov for advice on data processing. Assistance by the 08ID-1 beamline personnel at the Canadian Light Source is gratefully appreciated. Financial support for this research was provided by a grant from the Natural Sciences and Engineering Research Council of Canada awarded to YST and a grant (MOP-114889) from the Canadian Institutes of Health Research awarded to AMB. We also thank other funding agencies: JP is the recipient of a postdoctoral training award from the Fonds de Recherche Santé Québec, and AMB holds a Canada Research Chair in Structural Biology.

\section{References}

Aripirala, S., Szajnman, S. H., Jakoncic, J., Rodriguez, J. B., Docampo, R., Gabelli, S. B. \& Amzel, L. M. (2012). J. Med. Chem. 55, 6445-6454.

Bevington, A., Mundy, K. I., Yates, A. J., Kanis, J. A., Russell, R. G., Taylor, D. J., Rajagopalan, B. \& Radda, G. K. (1986). Clin. Sci. 71, 729-735.

Brennan, S. \& Cowan, P. L. (1992). Rev. Sci. Instrum. 63, 850.

Cao, R., Chen, C. K.-M., Guo, R.-T., Wang, A. H.-J. \& Oldfield, E. (2008). Proteins, 73, 431-439.

De Schutter, J. W., Shaw, J., Lin, Y.-S. \& Tsantrizos, Y. S. (2012). Bioorg. Med. Chem. 20, 5583-5591.

Emsley, P., Lohkamp, B., Scott, W. G. \& Cowtan, K. (2010). Acta Cryst. D66, 486-501.

Gabelli, S. B., McLellan, J. S., Montalvetti, A., Oldfield, E., Docampo, R. \& Amzel, L. M. (2006). Proteins, 62, 80-88.

Hol, W. G. J., van Duijnen, P. T. \& Berendsen, H. J. C. (1978). Nature (London), 273, 443-446.

Holloway, P. W. \& Popják, G. (1967). Biochem. J. 104, 57-70.

Huang, C. H., Gabelli, S. B., Oldfield, E. \& Amzel, L. M. (2010). Proteins, 78, 888-899.

Jahnke, W. et al. (2010). Nature Chem. Biol. 6, 660-666.

Kavanagh, K. L., Guo, K., Dunford, J. E., Wu, X., Knapp, S., Ebetino, F. H., Rogers, M. J., Russell, R. G. \& Oppermann, U. (2006). Proc. Natl Acad. Sci. USA, 103, 7829-7834.

Koul, H. K., Koul, S. \& Meacham, R. B. (2012). Prostate Cancer Prostatic Dis. 15, 111-119. 
Larkin, M. A., Blackshields, G., Brown, N. P., Chenna, R., McGettigan, P. A., McWilliam, H., Valentin, F., Wallace, I. M., Wilm, A., Lopez, R., Thompson, J. D., Gibson, T. J. \& Higgins, D. G. (2007). Bioinformatics, 23, 29472948.

Lin, Y.-S., Park, J., De Schutter, J. W., Huang, X. F., Berghuis, A. M., Sebag, M. \& Tsantrizos, Y. S. (2012). J. Med. Chem. 55, 3201-3215.

Mao, J., Mukherjee, S., Zhang, Y., Cao, R., Sanders, J. M., Song, Y., Zhang, Y., Meints, G. A., Gao, Y. G., Mukkamala, D., Hudock, M. P. \& Oldfield, E. (2006). J. Am. Chem. Soc. 128, 14485-14497.

McTaggart, S. J. (2006). Cell. Mol. Life Sci. 63, 255-267.

Mönkkönen, H., Kuokkanen, J., Holen, I., Evans, A., Lefley, D. V., Jauhiainen, M., Auriola, S. \& Mönkkönen, J. (2008). Anticancer Drugs, 19, 391-399.

Murshudov, G. N., Skubák, P., Lebedev, A. A., Pannu, N. S., Steiner, R. A., Nicholls, R. A., Winn, M. D., Long, F. \& Vagin, A. A. (2011). Acta Cryst. D67, 355-367.

Park, J., Lin, Y.-S., De Schutter, J. W., Tsantrizos, Y. S. \& Berghuis, A. M. (2012). BMC Struct. Biol. 12, 32.
Rondeau, J. M., Bitsch, F., Bourgier, E., Geiser, M., Hemmig, R., Kroemer, M., Lehmann, S., Ramage, P., Rieffel, S., Strauss, A., Green, J. R. \& Jahnke, W. (2006). ChemMedChem, 1, 267-273.

Sheldrick, G. M. (2010). Acta Cryst. D66, 479-485.

Spina, A., Sapio, L., Esposito, A., Di Maiolo, F., Sorvillo, L. \& Naviglio, S. (2013). Biores. Open Access, 2, 47-54.

Spina, A., Sorvillo, L., Di Maiolo, F., Esposito, A., D’Auria, R., Di Gesto, D., Chiosi, E. \& Naviglio, S. (2013). J. Cell. Physiol. 228, 198-206.

Tarshis, L. C., Yan, M., Poulter, C. D. \& Sacchettini, J. C. (1994). Biochemistry, 33, 10871-10877.

Thorn, A. \& Sheldrick, G. M. (2011). J. Appl. Cryst. 44, 1285-1287.

Winter, G., Lobley, C. M. C. \& Prince, S. M. (2013). Acta Cryst. D69, 12601273.

Zhang, Y. et al. (2009). J. Am. Chem. Soc. 131, 5153-5162.

Zhang, Y., Zhu, W., Liu, Y.-L., Wang, H., Wang, K., Li, K., No, J. H., Ayong, L., Gulati, A., Pang, R., Freitas-Junior, L., Morita, C. T. \& Oldfield, E. (2013). ACS Med. Chem. Lett. 4, 423-427. 